\title{
MINIMAX AND BAYES ESTIMATION IN DECONVOLUTION PROBLEM*
}

\author{
Mikhail ERMAKov ${ }^{1}$
}

\begin{abstract}
We consider a deconvolution problem of estimating a signal blurred with a random noise. The noise is assumed to be a stationary Gaussian process multiplied by a weight function function $\epsilon h$ where $h \in L_{2}\left(R^{1}\right)$ and $\epsilon$ is a small parameter. The underlying solution is assumed to be infinitely differentiable. For this model we find asymptotically minimax and Bayes estimators. In the case of solutions having finite number of derivatives similar results were obtained in [5].
\end{abstract}

Mathematics Subject Classification. 62G05, 65R30, 65R32.

Received September 12, 2007.

\section{INTRODUCTION AND MAIN RESULTS}

\subsection{Introduction}

We study the deconvolution problem in the following setting. We wish to estimate $x$ from the observed random process

$$
Y(t)=\int_{-\infty}^{\infty} a(t-s) x(s) \mathrm{d} s+\epsilon \eta(t), t \in R^{1} .
$$

Here the kernel $a$ is known and $\epsilon \eta(t)$ is a random noise with a small parameter $\epsilon>0$. Such a setting arises in many applications (see e.g. $[17,20,21,29]$ and references therein).

The noise $\epsilon \eta$ is usually considered as a stationary process. This allows to study the problem in terms of the Fourier transform and to derive the estimators in a simple analytic form. For this setting the statistical properties of Tikhonov regularizing algorithm, the procedure of Kolmogorov-Wiener filtration, robust and minimax estimators were analyzed in numerous publications and textbook (see, for example, $[10,21,29,31]$ and references therein). Recently interesting adaptive procedures (see $[2,3,14,30]$ ) and wavelet based estimators (see $[7,23,25]$ ) were proposed.

If the noise is a stationary process, the power of the noise $\epsilon^{2} \int_{-\infty}^{\infty} E \eta^{2}(t) \mathrm{d} t$ on the whole real line is infinite. Consistent estimator of solution $x(t)$ exists only if the ratio of the power of the noise to the power of the solution is finite. Thus, if we want to consider the estimation problem on real line, we need to suppose that the power of the solution is also infinite or tends to infinity. The assumptions of such a type became standard in the study of the Bayes and minimax estimators (see $[10,21,31])$. In practice the power of the solution is usually finite. Thus

\footnotetext{
Keywords and phrases. Deconvolution, minimax estimation, Bayes estimation, Wiener filtration.

* This paper was partially supported by RFFI Grants 02-01-00262, 4422.2006.1.

${ }^{1}$ Mechanical Engineering Problems Institute, Mechanical Engineering Problems Institute, Russian Academy of Sciences, Bolshoy pr. VO 61, 199178 St.Petersburg, Russia. 
it seems reasonable to consider modifications of the models with the finite power of solution on the whole real line. The study of such a modification is the goal of the paper.

The deconvolution problem is often considered in another setting as deconvolution problem on a circle (see $[8,18-20])$. In this model the powers of the noise and the solution are finite. However this setting requires the additional analysis of errors caused by such a model reduction.

In this paper we deal with the following noise model

$$
\epsilon \eta(t)=\epsilon h(t) \zeta(t)
$$

where $\zeta(t)$ is a stationary Gaussian process $\zeta(t), E \zeta(t)=0, E[\zeta(t) \zeta(0)]=r(t), t \in R^{1}$ and $h$ is a weight function $h \in L^{2}\left(R^{1}\right)$. The assumption $h \in L_{2}\left(R^{1}\right)$ implies that the noise power $\epsilon^{2} \int_{-\infty}^{\infty} E \eta^{2}(t) \mathrm{d} t=\epsilon^{2}\|h\|^{2} r(0)$ is finite. Thus we can assume that the power of solution is also finite.

From viewpoint of asymptotic statistics the model (1), (2) is closely related with another setting called often the problem of deconvolving a density (see $[2,3,6,12,13,25]$ ). In this setting we observe independent sample $Z_{1}=X_{1}+Y_{1}, \ldots, Z_{n}=X_{n}+Y_{n}$. A density function $a$ of i.i.d.r.v.'s $Y_{i}, 1 \leq i \leq n$ is known and a density function $x$ of i.i.d.r.v.'s $X_{1}, \ldots, X_{n}$ is unknown. We wish to estimate the density $x$.

Thus the density $x$ satisfies the convolution equation

$$
f(t)=(a * x)(t)=\int_{-\infty}^{\infty} a(t-s) x(s) \mathrm{d} s
$$

where $f(t)$ is the density of $Z_{1}, \ldots, Z_{n}$.

As it is well-known (see [24]) the problem of estimation of density $f$ is locally asymptotically equivalent to the estimation of function $f$ in the following white noise model

$$
\mathrm{d} Y(t)=f(t) \mathrm{d} t+\epsilon f_{0}^{1 / 2}(t) \mathrm{d} w(t)
$$

Here $\mathrm{d} w(t)$ stands for the Gaussian white noise and it is supposed that $f$ lies in the small vicinity of known function $f_{0}$ (see [24] for details).

We can rewrite (4) in the following form

$$
\mathrm{d} Y(t)=\int_{-\infty}^{\infty} a(t-s) x(s) \mathrm{d} s+\epsilon\left(\int_{-\infty}^{\infty} a(t-s) x_{0}(s) \mathrm{d} s\right)^{1 / 2} \mathrm{~d} w(t) .
$$

Here we suppose that $x$ lies in the small vicinity of known function $x_{0}(t), f_{0}=a * x_{0}$. If we put $h=\left(a * x_{0}\right)^{1 / 2}$, we get the paper model. The local asymptotic equivalence of the model () and the model of deconvolving a density has not been studied. However one can show that a wide class of linear estimators in the problem of deconvolving a density have the same asymptotic behaviour as in the model (5).

The model of estimation in the weighted Gaussian white noise arises also in the problems of asymptotic estimation of regression function (see [1]). As first publication on asymptotically minimax estimation in this model one can mention [9].

The goal of our paper is to derive asymptotically minimax and asymptotically Bayes estimators for the model $(1,2)$. We already considered this problem (see [11]) for the functions $x$ with a finite number of derivatives. In [11] we showed that asymptotically minimax and Bayes estimators have the same form as in the model with the Gaussian stationary noise (see $[10,31])$. This paper extend these results to the case of supersmooth functions. The models with supersmooth functions naturally arise in statistical applications (see $[4,5,28,30]$ ).

In $[4,5,30]$ the spectrum of operator corresponding to the kernel $a$ is discrete. In our model the operator spectrum is continuous. The operator corresponding to the function $h$ does not commute with the operators corresponding to the kernel $a$ and the correlation function $r$. These differences lead to implementation of serious analytical technique in the proof of the results. 
The model of deconvolving a density with supersmooth density $x$ is rather natural. The asymptotic behaviour of kernel estimators having minimax rates of convergence was analyzed in [12]. The adaptive minimax rates of convergence were studied in $[3,4]$. As the first publications on the estimation of supersmooth functions with the sharp minimax asymptotic one should mention $[10,15,16,27]$.

This paper can be considered as natural complement of [11]. The results of these papers cover the cases of the both smooth and supersmooth behaviour of kernel and solution. It turns out that, if the kernel or the solution is supersmooth, the asymptotically minimax and the asymptotically Bayes estimators are simple projection estimators. Only if both the solution and the kernel have finite smoothness, we are forced to define the estimators with the more complicated structure (see [11]). In this case the estimators based on the KolmogorovWiener filters are asymptotically Bayes (see $[21,31]$ ) and the standard minimax estimators are asymptotically $\operatorname{minimax}($ see $[8,10,26])$. The standard minimax estimators remain asymptotically minimax in the case of supersmooth kernel or solution. However in these cases we can define essentially more simple estimators with the same property.

For any function $z \in L_{2}\left(R^{1}\right)$ denote by

$$
Z(\omega)=\int \exp \{2 \pi i \omega t\} z(t) \mathrm{d} t
$$

the Fourier transform of $z$ and for any $z \in L_{2}\left(R^{1}\right)$ denote by

$$
\|z\|=\left(\int z^{2}(t) \mathrm{d} t\right)^{1 / 2}
$$

the $L_{2}$-norm of $z$. Hereafter the limits of integration are omitted if the integration domain is the real line $R^{1}$.

We suppose that the kernel $a$ satisfies A0,A1-A3 if the kernel $a$ has a finite number of derivatives and A0, A3, A4 in the case of supersmooth kernel.

A0. There holds $A(\omega)=A(-\omega)>0$ for all $\omega \in R^{1}$.

A1. There holds

$$
\lim _{t \rightarrow \infty} \int_{0}^{t} A^{-2}(\omega) R(\omega) \mathrm{d} \omega=\infty
$$

If the left-hand side of (6) is finite, the rate of convergence of estimators equals $\epsilon$.

A2. There exists $\gamma \geq 0$ such that for all $C>0$

$$
\lim _{\omega \rightarrow \infty} \frac{A(C \omega)}{A(\omega)}=C^{-\gamma}
$$

A3. There exists $C>0$ such that for all $\omega, \omega_{1} \in R^{1}$

$$
\left|A(\omega)-A\left(\omega_{1}\right)\right|<C\left|\omega-\omega_{1}\right| .
$$

A4. There exists $\gamma>0$ such that for all $C>0$

$$
\lim _{\omega \rightarrow \infty} \frac{\log A(C \omega)}{\log A(\omega)}=C^{\gamma} .
$$

If A2 holds, the kernel $a$ has a finite number of derivatives. If A4 holds, the kernel $a$ is infinitely differentiable.

The correlation function $r$ satisfies the following

R. There exists $\alpha>0$ such that for any $C>0$

$$
\lim _{\omega \rightarrow \infty} R(C \omega) / R(\omega)=C^{-\alpha} .
$$


If A2, $R$ hold with $\gamma=0, \alpha=0$ and $A(\omega) \equiv 1, R(\omega) \equiv 1$, we get the standard setting estimation of signal in the weighted Gaussian white noise

$$
\mathrm{d} y(t)=x(t) \mathrm{d} t+\epsilon h(t) \mathrm{d} w(t) .
$$

\subsection{Main results. Minimax estimation}

The assumption about the solution is rather standard (see $[8,10,26])$. We suppose that a priori information is given

$$
x \in Q=\left\{x: \int B^{2}(\omega)|X(\omega)|^{2} \mathrm{~d} \omega<1, x \in L_{2}\left(R^{1}\right)\right\}
$$

with the function $B$ satisfying the following.

B. The function $B(\omega)$ is even, positive and there exists $\beta>0$ such that for all $C>0$

$$
\lim _{\omega \rightarrow \infty} \frac{\ln B(C \omega)}{\ln B(\omega)}=C^{\beta} .
$$

Thus we have a priori information that the solution $x$ belongs to an ellipsoid in $L_{2}\left(R^{1}\right)$ and $\mathrm{B}$ implies that the solution $x(t)$ is infinitely differentiable.

The risk of any estimator $x^{*}(t)$ equals

$$
\rho_{\epsilon}\left(x^{*}\right)=\sup _{x \in Q} \int E\left(x^{*}(t)-x(t)\right)^{2} \mathrm{~d} t .
$$

We search for asymptotically minimax estimator $x_{\epsilon}^{* *}$ such that

$$
\rho_{\epsilon}=\rho_{\epsilon}\left(x_{\epsilon}^{* *}\right)=\inf _{x^{*}} \rho_{\epsilon}\left(x^{*}\right)(1+o(1)), \quad \epsilon \rightarrow 0 .
$$

Here the infimum over all estimators $x^{*}$.

We suppose that the function $h$ is smoother than the realizations of random process $\zeta(t)$ (see H2 below). Thus all information on the noise smoothness is contained in $\zeta(t)$.

H1. The function $H(\omega)$ is even, $H(\omega) \in L_{2}\left(R^{1}\right) \cap L_{1}\left(R^{1}\right)$ and $h(t)>0$ for all $t \in R^{1}$.

H2. There exists $\delta>0$ such that

$$
\lim _{\omega \rightarrow \infty} R^{-1}(\omega) H^{2}(\omega) \omega^{1+\delta}=0
$$

H3. $\int|t h(t)| \mathrm{d} t<\infty$.

Define the functions

$$
\Psi_{\epsilon}(\mu)=\epsilon^{2}\|h\|^{2} \int A^{-2}(\omega)(1-\mu B(\omega))_{+} R(\omega) \mathrm{d} \omega
$$

and

$$
\bar{\Psi}_{\epsilon}(\theta)=\epsilon^{2}\|h\|^{2} \int_{-\theta}^{\theta} A^{-2}(\omega) R(\omega) \mathrm{d} \omega .
$$

Hereafter $(u)_{+}=\max \{u, 0\}$ for all $u \in R^{1}$. We put $\omega_{1 \epsilon}=\sup \left\{\omega: \bar{\Psi}_{\epsilon}(\omega) \leq B^{-2}(\omega)\right\}$ and $\omega_{\epsilon}=\omega_{1 \epsilon}\left(1+\delta_{\epsilon}\right)$ where $\delta_{\epsilon}>0$ is such that $B\left(\omega_{1 \epsilon}\right)=o\left(B\left(\omega_{\epsilon}\right)\right), A^{-1}\left(\omega_{1 \epsilon}\right)=A^{-1}\left(\omega_{\epsilon}\right)(1+o(1))$ and $\delta_{\epsilon} \rightarrow 0$ as $\epsilon \rightarrow 0$. Denote $\mu_{\epsilon}=\sup \left\{\mu: B\left(\omega_{\epsilon}\right)>\mu\right\}$.

Define the kernels

and

$$
K_{\mu_{\epsilon}}(\omega)=A^{-1}(\omega)\left(1-\mu_{\epsilon} B(\omega)\right)_{+}
$$

$$
K_{\omega_{\epsilon}}(\omega)=A^{-1}(\omega) \chi\left(|\omega|<\omega_{\epsilon}\right) .
$$


Hereafter $\chi(U)$ denotes the indicator of event $U$. The kernel $k_{\mu_{\epsilon}}$ is the kernel of standard asymptotically minimax estimator $x_{\mu_{\epsilon}}^{* *}(t)=\left(k_{\mu_{\epsilon}} * y\right)(t)$ (see [8-11,26]). The kernel $k_{\omega_{\epsilon}}$ is the kernel of simple projection estimator $x_{\omega_{\epsilon}}^{* *}(t, y)=\left(k_{\omega_{\epsilon}} * y\right)(t)$.

Define also the kernels $k_{\alpha}$ of Tikhonov regularizing algorithm

$$
K_{\alpha}(\omega)=\frac{A(\omega)}{A^{2}(\omega)+\alpha M(\omega)}
$$

where the function $M(\omega)$ satisfies the following.

M1. The function $M(\omega)$ is even, nonnegative and increasing in $R_{+}^{1}$.

M2. The function $M(\omega)\left|B^{-1}(\omega) A^{-2}(\omega)\right|$ is nondecreasing in $R_{+}^{1}$.

M2 implies that the function $M(\omega)$ has the exponential growth. The exponential growth of function $M(\omega)$ in Tikhonov regularizing algorithm is natural assumption in the case of a priori information on supersmooth solution (see [20]).

Define the parameters of regularization $\alpha_{\epsilon}=A^{2}\left(\omega_{\epsilon}\right) M^{-1}\left(\omega_{\epsilon}\right)$.

If the solution $x$ is smoother than the kernel $a$ (A2 or A4 with $\beta>\gamma$ holds) the asymptotic of minimax risks is attained for all estimators mentioned above: for the standard minimax estimator $k_{\mu_{e}}$ (see (9)), for the simple projection estimator $k_{\omega_{\epsilon}}$ (see (10)) and the estimator of Tikhonov regularizing algorithm (see (11)). If the kernel $a$ is smoother then the solution $x$ (A4, $B$ holds with $\gamma>\beta$ ), then the lower bound of asymptotic of minimax risks is attained only in the cases of simple projection estimator and estimator of Tikhonov regularizing algorithm. These statements are given in Theorems 1 and 2 respectively. In both theorems we assume that $h$ is smoother than the realizations of random process $\zeta(t)$ (see $\mathrm{H} 2$ above).

Theorem 1.1. Assume A0-A3, B, H1-H3, $R$ or A0, A3, A4, B, H1-H3, $R$ with $\beta>\gamma$. Then the estimators $x_{\mu_{\epsilon}}^{* *}(t)=\left(k_{\mu_{\epsilon}} * y\right)(t)$ and $x_{\omega_{\epsilon} *}^{* *}(t, y)=\left(k_{\omega_{\epsilon}} * y\right)(t)$ are asymptotically minimax. The asymptotically minimax risks equal

$$
\rho_{\epsilon}\left(x_{\mu_{\epsilon}}^{* *}\right)=\rho_{\epsilon}\left(x_{\omega_{\epsilon}}^{* *}\right)(1+o(1))=\Psi_{\epsilon}\left(\mu_{\epsilon}\right)(1+o(1))=\bar{\Psi}_{\epsilon}\left(\omega_{\epsilon}\right)(1+o(1))=\bar{\Psi}_{\epsilon}\left(\omega_{1 \epsilon}\right)(1+o(1)) .
$$

If M1,M2 hold also, the Tikhonov regularizing algorithm $x_{\alpha_{\epsilon}}^{* *}=k_{\alpha_{\epsilon}} * y$ is asymptotically minimax.

The presence of $\|h\|^{2}$ in the asymptotic $\Psi_{\epsilon}\left(\mu_{\epsilon}\right)$ of asymptotically minimax risk $\rho_{\epsilon}$ and in the definition (9) of asymptotically minimax estimator $x_{\mu_{e}}^{* *}$ is the unique difference from the standard definitions of asymptotically minimax risks and asymptotically minimax estimator (see $[8-11,26])$.

Remark 1. Denote $\Lambda$ the set of functions $h$ satisfying $\mathrm{H} 1, \mathrm{H} 3$ and such that the convergence in $\mathrm{H} 2$ is uniform w.r.t. all $h \in \Lambda$. Suppose also that there exist functions $h_{01}, h_{0} \in \Lambda$ such that $h_{01} \geq h(t) \geq h_{0}(t)>0, t \in R^{1}$ for all $h \in \Lambda$. Then the asymptotics of minimax risks $\rho_{\epsilon h}\left(x_{\mu_{\epsilon}}^{* *}\right)=\rho_{\epsilon h}\left(x_{\omega_{\epsilon}}^{* *}\right)(1+o(1))$ are uniform with respect to $h \in \Lambda$. Moreover all information on $h$ in the estimators $x_{\omega_{\epsilon}}^{* *}, x_{\mu_{\epsilon}}^{* *}$ is contained in $\|h\|$. Thus we can consider $h$ as unknown in the model supposing only $h \in \Lambda$. Naturally, since $\|h\|$ is unknown, the problems of the choice of regularization parameters $\mu_{\epsilon}$ and $\omega_{\epsilon}$ arise. However, since other parameters of models are usually unknown as well (for example $R(\omega), B(\omega)$ ), these problems arise also on other reasons. Note that similar remark on the uniform risks convergence hold for the other theorems of this paper and for theorems of [11] as well.

If the kernel $a$ is smoother than the solution $x$ and $\gamma>\beta$ (see A3, B), the projection estimator $x_{\omega_{\epsilon}}^{* *}=k_{\omega_{\epsilon}} * y$ and the Tikhonov regularizing algorithm $x_{\alpha_{\epsilon}}^{* *}=k_{\alpha_{\epsilon}} * y$ remain asymptotically minimax. However the formula for asymptotic of minimax risks is defined in another form.

Let $\omega_{1 \epsilon}$ satisfy the equation

$$
B^{-2}\left(\omega_{1 \epsilon}\right)=\epsilon^{2}\|h\|^{2} \int_{-\omega_{1 \epsilon}}^{\omega_{1 \epsilon}} A^{-2}(\omega) R(\omega) \mathrm{d} \omega .
$$

Define $\delta_{\epsilon}>0$ such that $\delta_{\epsilon}=o\left(\omega_{1 \epsilon}^{-\beta}\right)$ and $\omega_{1 \epsilon}^{-\gamma}=o\left(\delta_{\epsilon}\right)$. Denote $\omega_{\epsilon}=\left(1-\delta_{\epsilon}\right) \omega_{1 \epsilon}$ and $\alpha_{\epsilon}=A^{2}\left(\omega_{\epsilon}\right) M^{-1}\left(\omega_{\epsilon}\right)$. 
Theorem 1.2. Assume A0, A4, B, H1, H2 and R. Let $\gamma>\beta$. Then the family of projection estimators $x_{\omega_{\epsilon}}^{* *}(t, y)=\left(k_{\omega_{\epsilon}} * y\right)(t)$ is asymptotically minimax. There holds

$$
\rho_{\epsilon}\left(x_{\omega_{\epsilon}}^{* *}\right)=B^{-2}\left(\omega_{\epsilon}\right)(1+o(1))=B^{-2}\left(\omega_{1 \epsilon}\right)(1+o(1)) .
$$

If $M 1$ holds, the Tikhonov regularizing algorithm $x_{\alpha_{\epsilon}}^{* *}=k_{\alpha_{\epsilon}} * y$ is also asymptotically minimax.

Example 1. Let

$$
\begin{aligned}
& A(\omega)=A_{1}(\omega)|\omega|^{-\gamma}, \\
& B(\omega)=B_{2} \exp \left\{B_{1}(\omega)|\omega|^{\beta}\right\} \\
& R(\omega)=R_{1}(\omega)|\omega|^{-\alpha}
\end{aligned}
$$

and let the assumptions of Theorem 1.1 hold.

Let $\omega_{1 \epsilon}$ be defined the equation

$$
B_{1}\left(\omega_{1 \epsilon}\right) \omega_{1 \epsilon}^{\beta}=|\ln \epsilon|
$$

Then one can put $\omega_{\epsilon}=\omega_{1 \epsilon}\left(1+\delta_{\epsilon}\right)$ where $\delta_{\epsilon}>0, \delta_{\epsilon}|\ln \epsilon|^{1-\kappa} \rightarrow \infty, \delta_{\epsilon} \rightarrow 0$ as $\epsilon \rightarrow 0$ with $1>\kappa>0$ and

$$
\rho_{\epsilon}=\frac{2\|h\|^{2}}{2 \gamma-\alpha+1} \epsilon^{2} \omega_{1 \epsilon}^{2 \gamma-\alpha+1} A_{1}^{-2}\left(\omega_{1 \epsilon}\right) R_{1}\left(\omega_{1 \epsilon}\right)(1+o(1)) .
$$

If $A_{1}(\omega)=A_{1}, B_{1}(\omega)=B_{1}, R_{1}(\omega)=R_{1}$ are constants, we get

$$
\rho_{\epsilon}=A_{1}^{-2} R_{1} B_{1}^{\frac{\alpha-2 \gamma-1}{\beta}} \frac{2\|h\|^{2}}{2 \gamma-\alpha+1} \epsilon^{2}|\ln \epsilon|^{\frac{2 \gamma-\alpha+1}{\beta}}(1+o(1)) .
$$

In the case of standard setting signal detection in the Gaussian white noise $(A(\omega) \equiv 1, R(\omega) \equiv 1)$

$$
\rho_{\epsilon}=2 B_{1}^{-\frac{1}{\beta}}|| h \|^{2} \epsilon^{2}|\ln \epsilon|^{\frac{1}{\beta}}(1+o(1)) .
$$

Example 2. Let

$$
A(\omega)=A_{2} \exp \left\{-A_{1}(\omega)|\omega|^{\gamma}\right\}
$$

and (16), (17) hold with $\beta>2 \gamma$. Then we can put $\omega_{\epsilon}=\omega_{1 \epsilon}\left(1+\delta_{\epsilon}\right)$ where $\omega_{1 \epsilon}$ is defined (18) and $\delta_{\epsilon}>$ $0, \delta_{\epsilon}|\ln \epsilon|^{1-\gamma / \beta-\kappa} \rightarrow \infty, \delta_{\epsilon} \rightarrow 0$ as $\epsilon \rightarrow 0$ with $1-\gamma / \beta>\kappa>0$. The asymptotically minimax risk equals and

$$
\rho_{\epsilon}=\frac{2}{\gamma} A_{2}^{-2}\|h\|^{2} \epsilon^{2} \omega_{1 \epsilon}^{1-\gamma-\alpha} R_{1}\left(\omega_{1 \epsilon}\right) \exp \left\{2 A_{1}\left(\omega_{1 \epsilon}\right)\left|\omega_{1 \epsilon}\right|^{\gamma}\right\}(1+o(1)) .
$$

If $A_{1}(\omega)=A_{1}, B_{1}(\omega)=B_{1}, R_{1}(\omega)=R_{1}$ are constants, we get

$$
\left.\rho_{\epsilon}=\left.\left.\frac{2}{\gamma} A_{2}^{-2} B_{1}^{\frac{\alpha+\gamma-1}{\beta}} R_{1}|| h\right|^{2} \epsilon^{2}|\ln \epsilon|^{\frac{1-\gamma-\alpha}{\beta}} \exp \left\{2 A_{1} B_{1}^{-\frac{\gamma}{\beta}} \mid \ln \epsilon\right)\right|^{\frac{\gamma}{\beta}}\right\}(1+o(1)) .
$$

Example 3. Let (16), (17), (21) hold with $2 \gamma \geq \beta>\frac{3}{2} \gamma$ and let $A_{1}(\omega)=A_{1}, B_{1}(\omega)=B_{1}, R_{1}(\omega)=R_{1}$ be constants. Then

and

$$
\omega_{1 \epsilon}=B_{1}^{-1 / \beta}|\ln \epsilon|^{1 / \beta}\left(1-\frac{1}{\beta} A_{1} B_{1}^{-\gamma / \beta}|\ln \epsilon|^{\frac{\gamma}{\beta}-1}\right)
$$

$$
\begin{aligned}
\rho_{\epsilon}= & \gamma^{-1} A_{1}^{-1} A_{2}^{-2} B_{1}^{\frac{\alpha+\gamma-1}{\beta}} R_{1} \|\left. h\right|^{2} \epsilon^{2}|\ln \epsilon|^{\frac{1-\gamma-\alpha}{\beta}} \\
& \times \exp \left\{2 A_{1} B_{1}^{-\frac{\gamma}{\beta}}|\ln \epsilon|^{\frac{\gamma}{\beta}}\left(1-\frac{\gamma}{\beta} A_{1} B_{1}^{-\frac{\gamma}{\beta}}|\ln \epsilon|^{\frac{\gamma}{\beta}-1}\right)\right\}(1+o(1)) .
\end{aligned}
$$


Example 4. Let (16), (17), (21) hold with $\gamma>2 \beta$. Define $\omega_{1 \epsilon}$ the equation

$$
A_{1}\left(\omega_{1 \epsilon}\right) \omega_{1 \epsilon}^{\gamma}=|\ln \epsilon|
$$

and put $\omega_{\epsilon}=\omega_{1 \epsilon}\left(1-\delta_{\epsilon}\right)$ where $\delta_{\epsilon}>0, \delta_{\epsilon} \rightarrow 0, \delta_{\epsilon}|\ln \epsilon|^{1-\beta / \gamma-\kappa} \rightarrow \infty$ as $\epsilon \rightarrow 0$ with $0<\kappa<1-\beta / \gamma$. Then

$$
\rho_{\epsilon}=B_{2}^{2} \exp \left\{-2 B_{1}\left(\omega_{1 \epsilon}\right) \omega_{1 \epsilon}^{\beta}\right\}(1+o(1)) .
$$

If $A_{1}(\omega)=A_{1}, B_{1}(\omega)=B_{1}$ are constants, we get

$$
\rho_{\epsilon}=B_{2}^{2} \exp \left\{-2 B_{1} A_{1}^{-\frac{\beta}{\gamma}}|\ln \epsilon|^{\frac{\beta}{\gamma}}\right\}(1+o(1)) .
$$

Example 5. Let (16), (17), (21) hold with $2 \beta \geq \gamma>3 / 2 \beta$ and let $A_{1}(\omega)=A_{1}, B_{1}(\omega)=B_{1}, R_{1}(\omega)=R_{1}$ be constants. Then

$$
\omega_{1 \epsilon}=A_{1}^{-1 / \gamma}|\ln \epsilon|^{1 / \gamma}\left(1-\frac{1}{\gamma} B_{1} A_{1}^{-\beta / \gamma}|\ln \epsilon|^{\frac{\beta}{\gamma}-1}\right)
$$

and

$$
\rho_{\epsilon}=B_{2}^{2} \exp \left\{-2 A_{1}^{-\frac{\beta}{\gamma}} B_{1}|\ln \epsilon|^{\frac{\beta}{\gamma}}\left(1-\frac{\beta}{\gamma} A_{1}^{-\frac{\beta}{\gamma}} B_{1}|\ln \epsilon|^{\frac{\beta}{\gamma}-1}\right)\right\}(1+o(1)) .
$$

\subsection{Main results. Bayes approach}

In the Bayes setting we suppose that the solution $x$ is a realization of the random process

$$
x(t)=h_{1}(t) \xi(t)
$$

where $h_{1}(t) \in L_{2}\left(R^{1}\right)$ and $\xi$ is Gaussian stationary random process, $E \xi(t)=0, E[\xi(t) \xi(0)]=v(t), t \in R^{1}$.

As follows from assumption $\mathrm{V}$ given bellow we suppose that the realizations of random process $\xi(t)$ are infinitely differentiable.

V. There exists $\beta>0$ such that for all $C>0$ there holds

$$
\lim _{\omega \rightarrow \infty} \frac{\ln V(C \omega)}{\ln V(\omega)}=C^{\beta}
$$

The function $h_{1}(t)$ satisfies the following assumptions.

H4. The function $h_{1}(t)$ is even, bounded and $h_{1}(t)>\operatorname{ch}(t)>0$ for all $t \in R^{1}$ with constant $c>0$.

H5. There holds

$$
\lim _{\omega \rightarrow \infty} \frac{\ln H_{1}(\omega)}{\ln V(\omega)}=\infty
$$

H5 implies that the main information on the smoothness of $x$ is contained in the random process $\xi(t)$.

For any estimator $x^{*}$ define the Bayes risk

$$
\hat{\rho}_{\epsilon}\left(x^{*}\right)=E_{\xi} E_{\zeta}\left\|x^{*}-h_{1} \xi\right\|^{2} .
$$

We say that the estimator $\bar{x}_{\epsilon}^{*}$ is asymptotically Bayes if

$$
\hat{\rho}_{\epsilon}=\hat{\rho}_{\epsilon}\left(\bar{x}_{\epsilon}^{*}\right)=\inf _{x^{*}} \hat{\rho}_{\epsilon}\left(x^{*}\right)(1+o(1)), \quad \epsilon \rightarrow 0 .
$$

Here the infimum is over all estimator $x^{*}$. 
In this setting we could not prove that the Wiener filters

$$
K_{\epsilon}(\omega)=\left\|h_{1}\right\|^{2} A(\omega) V(\omega)\left(\left\|h_{1}\right\|^{2} A^{2}(\omega) V(\omega)+\epsilon^{2}\|h\|^{2} R(\omega)\right)^{-1}
$$

are asymptotically Bayes. At the same time we show that the simple projection estimator $x_{\omega_{\epsilon}}^{* *}$ are asymptotically Bayes. The value of $\omega_{\epsilon}$ is defined by the equation $\omega_{\epsilon}=\omega_{1 \epsilon}\left(1+\delta_{\epsilon}\right)$ where $\omega_{1 \epsilon}$ satisfies the equation

$$
A^{2}\left(\omega_{1 \epsilon}\right)\left\|h_{1}\right\|^{2} V\left(\omega_{1 \epsilon}\right)=\epsilon^{2}\|h\|^{2} R\left(\omega_{1 \epsilon}\right),
$$

and $\delta_{\epsilon}>0, \delta_{\epsilon} \rightarrow 0$ as $\epsilon \rightarrow 0$, is such that

$$
V\left(\omega_{\epsilon}\right)=o\left(V\left(\omega_{1 \epsilon}\right)\right), \quad H_{1}^{2}\left(\frac{1}{2} \delta_{\epsilon} \omega_{1 \epsilon}\right)=o\left(\epsilon^{2} A^{-2}\left(\omega_{\epsilon}\right) R\left(\omega_{\epsilon}\right)\right) .
$$

Theorem 1.3. Assume A0, A1, A2, H1, H2, H4, H5, R, V. Then the family of estimators $x_{\omega_{\epsilon}}^{* *}=\left(k_{\omega_{\epsilon}} * y\right)(t)$ is asymptotically Bayes. There holds

$$
\hat{\rho}_{\epsilon}\left(x_{\omega_{\epsilon}}^{* *}\right)=\epsilon^{2}\|h\|^{2} \int_{-\omega_{1 \epsilon}}^{\omega_{1 \epsilon}} A^{-2}(\omega) R(\omega) \mathrm{d} \omega(1+o(1)) .
$$

Note that $\omega_{1 \epsilon}$ in (31) can be replaced by $\omega_{\epsilon}$.

\section{Example 6. Let}

and (15), (17) hold. Then

$$
V(\omega)=\exp \left\{-V_{1}|\omega|^{\beta}\right\}
$$

$$
\omega_{1 \epsilon}=V_{1}^{1 / \beta}|\ln \epsilon|^{1 / \beta}
$$

and $\omega_{\epsilon}=\omega_{1 \epsilon}\left(1+\delta_{\epsilon}\right)$ where $\delta_{\epsilon}>0, \delta_{\epsilon} \rightarrow 0, \delta_{\epsilon}|\ln \epsilon|^{1-\kappa} \rightarrow \infty$ as $\epsilon \rightarrow 0$ with $0<\kappa<1$.

We have

$$
\hat{\rho}_{\epsilon}=\frac{2\|h\|^{2}}{2 \gamma-\alpha+1} V_{1}^{\frac{2 \gamma-\alpha+1}{\beta}} \epsilon^{2}|\ln \epsilon|^{\frac{2 \gamma-\alpha+1}{\beta}} A_{1}^{-2}\left(\omega_{\epsilon}\right) R_{1}\left(\omega_{\epsilon}\right)(1+o(1)) .
$$

For the model of signal detection in Gaussian white noise $A(\omega) \equiv 1, R(\omega) \equiv 1$ we get

$$
\hat{\rho}_{\epsilon}=2\|h\|^{2} V_{1}^{\frac{1}{\beta}} \epsilon^{2}|\ln \epsilon|^{\frac{1}{\beta}}(1+o(1)) .
$$

Remark 2. In applications the kernel $a$ is often unknown and the information about the kernel can be obtained only from statistical experiment (see [21,29]). As a consequence, the arising estimator $\hat{a}$ of kernel $a$ is known with a random error. Usually it is supposed that the error admits the Gaussian approximation. If we study the quality of estimation of solution $x$ one needs to find the influence of this random error on the risk function.

We consider the following model of kernel estimator

$$
\hat{a}(t)=a(t) \mathrm{d} t+\kappa_{\epsilon} \bar{h}(t) \mathrm{d} w(t)
$$

with the function $\bar{h} \in L_{2}\left(R^{1}\right)$ satisfying the following

$$
\begin{gathered}
\int t^{2} \bar{h}^{2}(t) \mathrm{d} t<\infty, \\
\lim _{\omega \rightarrow \infty} \bar{H}^{2}(\omega)|\omega|^{1+\delta}=0
\end{gathered}
$$

where $\delta>0$.

The Fourier transform of $\hat{a}$ can be written in the following form

$$
\hat{A}(\omega)=A(\omega)+\kappa_{\epsilon} \hat{\tau}(\omega)
$$


where

$$
\hat{\tau}(\omega)=\int \bar{H}\left(\omega-\omega_{1}\right) d w\left(\omega_{1}\right)
$$

is Gaussian stationary process.

Straightforward calculations show that, if $M_{\epsilon} \doteq \sup \left\{|\hat{\tau}(\omega),| \omega \mid \leq \omega_{\epsilon}\right\}=o_{P}\left(A\left(\omega_{\epsilon}\right) / \kappa_{\epsilon}\right)$, the random error $\kappa_{\epsilon} \hat{\tau}(\omega)$ does not influence on the asymptotic of minimax and Bayes risks and the estimators $x_{\omega_{\epsilon}}^{* *}$ remains asymptotically minimax and asymptotically Bayes. The reasoning is the following.

If (32),(33) holds, by Theorem 12.3.5 in [22],

$$
\lim _{\epsilon \rightarrow 0} P\left(\|h\|^{-1}\left(2 \ln \left(2 \omega_{\epsilon}\right)\right)^{-1 / 2}\left|M_{\epsilon}-\|h\|\left(2 \ln \left(2 \omega_{\epsilon}\right)\right)^{1 / 2}\right|>u\right)=\exp \{-C \exp \{-u\}\} .
$$

Hence, if $\kappa_{\epsilon}=o\left(A\left(\omega_{\epsilon}\right)\left|\ln \omega_{\epsilon}\right|^{1 / 2}\right)$, the influence of noise $\kappa_{\epsilon} \bar{h}(t) d w(t)$ on the kernel $a$ is negligible and the asymptotic of risks for the estimator $x_{\omega_{\epsilon}}^{* *}$ are the same both in the minimax setting of Theorems 1.1, 1.2 and the Bayes setting of Theorem 1.3. A similar statements hold also for the asymptotically minimax settings Theorems 1.1 and 1.2 in [11]. Note that $\epsilon=o\left(A\left(\omega_{\epsilon}\right)\left(\ln \omega_{\epsilon}\right)^{1 / 2}\right)$. Thus we can estimate the solution $x$ in the case of random kernel with the same risk asymptotic even if the kernel estimator $\hat{a}$ have a larger error than $\epsilon$.

\section{Proofs of theorems}

\subsection{Proof of lower bound in Theorem 1.1}

The proof of lower bounds in minimax setting is based traditionally on the fact that the Bayes risk does not exceed the minimax one. We define such Bayes a priori distributions that the powers of realizations $\int_{\omega}^{\omega+\Delta \omega} X^{2}(u) \mathrm{d} u$ on each interval $(\omega, \omega+\Delta \omega) \subset \Omega_{\epsilon}=\left(-\omega_{1 \epsilon}\left(1-\delta_{\epsilon}\right), \omega_{1 \epsilon}\left(1-\delta_{\epsilon}\right)\right)$ have the large order then corresponding power of noise $\epsilon^{2}\|h\|^{2} \int_{\omega}^{\omega+\Delta \omega} A^{-2}(u) R(u) \mathrm{d} u$. Hence the bias of Bayes estimator does not influence on the asymptotic of Bayes risks. Thus we get that the asymptotic of Bayes risks is defined the random noise and the contribution of each interval $(\omega, \omega+\Delta \omega) \subset \Omega_{\epsilon}$ in the Bayes risk equals $\epsilon^{2}\|h\|^{2} \int_{\omega}^{\omega+\Delta \omega} A^{-2}(u) R(u) \mathrm{d} u(1+$ $o(1))$. This allows to choose $A^{-1}(\omega) Y(\omega)$ as asymptotically Bayes estimators $X(\omega)$ in $\Omega_{\epsilon}$ and to get the lower bound (12).

We put $\omega_{2 \epsilon}=\left(1-\delta_{\epsilon}\right) \omega_{1 \epsilon}$ where $\delta_{\epsilon}>0$ is such that $A\left(\omega_{2 \epsilon}\right)=A\left(\omega_{\epsilon}\right)(1+o(1))$,

$$
B^{-1}\left(\omega_{1 \epsilon}\right)=o\left(B^{-1}\left(\omega_{2 \epsilon}\right)\right), \quad \epsilon^{2} A^{-2}\left(\omega_{\epsilon}\right)\left(\omega_{\epsilon}^{1+\gamma+\beta} R^{-1}\left(\omega_{\epsilon}\right)+\omega_{\epsilon}^{3}\right)=o\left(B^{-2}\left(\omega_{2 \epsilon}\right)\right)
$$

and $\delta_{\epsilon} \rightarrow 0$ as $\epsilon \rightarrow 0$.

Define the values $D_{\epsilon}$ and $\Delta_{\epsilon}$ such that

$$
D_{\epsilon}=o\left(B^{-1}\left(\omega_{2 \epsilon}\right) \omega_{\epsilon}^{(1-\beta) / 2}\right), \quad \epsilon^{2} A^{-2}\left(\omega_{\epsilon}\right)\left(\omega_{\epsilon}^{1+\gamma+\beta} R^{-1}\left(\omega_{\epsilon}\right)+\omega_{\epsilon}^{3}\right)=o\left(D_{\epsilon}^{2}\right)
$$

and

$$
\Delta=\Delta_{\epsilon}=o\left(\epsilon^{2} D_{\epsilon}^{-2} R\left(\omega_{\epsilon}\right) \omega_{\epsilon}^{-\gamma}+\omega_{\epsilon}^{-3 / 2-\gamma} R\left(\omega_{\epsilon}\right)+\omega_{\epsilon}^{1-\beta}\right) .
$$

The Bayes a priori measures $\lambda_{\epsilon}=\lambda_{\epsilon} \Delta_{\epsilon}$ are defined as the conditional probability measures of Gaussian random processes $\xi_{\epsilon}=\xi_{\epsilon \Delta_{\epsilon}}$ under the condition $\xi_{\epsilon} \in Q$ where $Q$ is the set of all solution $x$ (see (8)). The random process $\xi_{\epsilon}=\xi_{\epsilon \Delta_{\epsilon}}$ is defined via the Fourier transform

$$
\hat{\xi}_{\epsilon}(\omega)=\xi_{\epsilon \Delta_{\epsilon}}=D_{\epsilon} \sum_{i=-l}^{l} \eta_{i} \chi\left(\omega \in I_{i}\right)
$$

where $l=l_{\epsilon}=\left[\omega_{2 \epsilon} / \Delta_{\epsilon}\right], I_{i}=\left((i-1 / 2) \Delta_{\epsilon},(i+1 / 2) \Delta_{\epsilon}\right)$ and $\eta_{i}$ are independent Gaussian random variables, $E \eta_{i}=0, E \eta_{i}^{2}=1,-l \leq i \leq l$. Hereafter $[t]$ denotes the integer part of $t \in R^{1}$. 
On the base of the partition on intervals $I_{i},-l \leq i \leq l$, we reduce the problem to the discrete version and show that the discrete version of the problem has a simple solution. The choice of $D_{\epsilon}$ satisfying (35) allows to get $\epsilon^{2} E \int_{I_{i}} \hat{\eta}^{2}(u) \mathrm{d} u=o\left(E \int_{I_{i}} \hat{\xi}_{\epsilon}^{2}(u) \mathrm{d} u\right)$ on all intervals $I_{i},|i|>\delta l, 0<\delta<1$. The variation of function $R(\omega)$ on interval $I_{i}$ is neglected, that is $R(\omega)=R\left(i \Delta_{\epsilon}\right)(1+o(1))$ if $\Delta$ satisfies (36). At the same time

$$
\int_{I_{i}} h^{2}(\omega-u) \mathrm{d} u=\|h\|^{2}(1+o(1))
$$

if $|\omega|<\left(1-\delta_{1}\right) \Delta, 0<\delta_{1}<1$. This allows to show that asymptotically minimax risks $\rho_{\epsilon}$ and asymptotically minimax estimators $x_{\mu_{\epsilon}}^{* *}, x_{\omega_{\epsilon}}^{* *}$ depend only on $\|h\|^{2}$.

Denote $\nu_{\epsilon}$ the probability measure of $\xi_{\epsilon}$.

Lemma 2.1. There holds

$$
\lim _{\epsilon \rightarrow 0} P\left(\xi_{\epsilon} \in Q\right)=1
$$

Proof. By straightforward calculations, we get

$$
\begin{array}{r}
E\left[\int B^{2}(\omega) \hat{\xi}_{\epsilon}^{2}(\omega) \mathrm{d} \omega\right]=O\left(D_{\epsilon}^{2} \int_{-\omega_{2 \epsilon}}^{\omega_{2 \epsilon}} B^{2}(\omega) \mathrm{d} \omega\right)=O\left(D_{\epsilon}^{2} B^{2}\left(\omega_{2 \epsilon}\right) \omega_{\epsilon}^{\beta-1}\right)=o(1), \\
\operatorname{Var}\left[\int B^{2}(\omega) \hat{\xi}_{\epsilon}^{2}(\omega) \mathrm{d} \omega\right]=O\left(D_{\epsilon}^{4} \int_{-\omega_{2 \epsilon}}^{\omega_{2 \epsilon}} B^{4}(\omega) \mathrm{d} \omega\right)=O\left(D_{\epsilon}^{4} B^{4}\left(\omega_{2 \epsilon}\right) \omega_{\epsilon}^{1-\beta}\right)=o\left(D_{\epsilon}^{4} B^{4}\left(\omega_{2 \epsilon}\right) \omega_{\epsilon}^{2-2 \beta}\right)
\end{array}
$$

as $\epsilon \rightarrow 0$. Hence, by Chebyshev inequality, using (35), we get (38). This completes the proof of Lemma 2.1 .

For any estimator $x_{\epsilon}^{*}$ define the Bayes risks

$$
\begin{aligned}
& \hat{\rho}_{\epsilon \lambda}\left(x_{\epsilon}^{*}\right)=\int_{Q} \mathrm{~d} \lambda_{\epsilon}(x) E\left\|x_{\epsilon}^{*}-x\right\|^{2}, \\
& \hat{\rho}_{\epsilon \nu}\left(x_{\epsilon}^{*}\right)=\int_{Q} \mathrm{~d} \nu_{\epsilon}(x) E\left\|x_{\epsilon}^{*}-x\right\|^{2} .
\end{aligned}
$$

Denote $\bar{x}_{\epsilon}^{*}$ and $\bar{x}_{\epsilon}$ the Bayes estimators corresponding to a priori measures $\lambda_{\epsilon}$ and $\nu_{\epsilon}$ respectively.

Lemma 2.2. There holds

$$
\hat{\rho}_{\epsilon \lambda}\left(\bar{x}_{\epsilon}^{*}\right) \geq \hat{\rho}_{\epsilon \nu}\left(\bar{x}_{\epsilon}\right)(1+o(1)), \quad \epsilon \rightarrow 0 .
$$

The proof of Lemma 2.2 is akin to that of Lemma 2.3 in [11] and is omitted.

By Lemmas 2.1 and 2.2, the proof of lower bound is reduced to the problem of obtaining asymptotic of Bayes risks $\hat{\rho}_{\epsilon \nu}\left(\bar{x}_{\epsilon}\right)$. Since a priori measures $\nu_{\epsilon}$ are Gaussian the analytic formula for the Bayes risks $\hat{\rho}_{\epsilon \nu}\left(\bar{x}_{\epsilon}\right)$ can be written straightforwardly (see (47) below). However this formula is rather cumbersome and the proof that the asymptotic of $\hat{\rho}_{\epsilon \nu}\left(\bar{x}_{\epsilon}\right)$ coincides with $\bar{\Psi}_{\epsilon}\left(\omega_{\epsilon}\right)$ requires special analytic technique.

Since the random process $\xi_{\epsilon}$ is Gaussian the Bayes estimator is linear

$$
\bar{x}_{\epsilon}=\int k_{\epsilon}(t, s) y(s) \mathrm{d} s
$$

with the kernel $k_{\epsilon}$ satisfying the equation (see (2.16) in [11])

$$
E_{\tau}\left[\bar{x}_{\epsilon}(t) f(s)\right]-E_{\tau}\left[\int k_{\epsilon}(t, u) f(u) \mathrm{d} u f(s)\right]-\epsilon^{2} \int k_{\epsilon}(t, u) h(s) h(u) r(u-s) \mathrm{d} u=0
$$


for all $t, s \in R^{1}$. Hereafter $f=a * x$. Note that (39) is obtained as the Gateaux derivative of the risk function

$$
E_{\xi_{\epsilon}} E_{\zeta}\left\|\int k(t, u) y(u) \mathrm{d} u-\xi_{\epsilon}\right\|^{2}
$$

In terms of Fourier transform (39) can be written as follows

$$
\begin{aligned}
V_{\Delta}\left(\omega, \omega_{0}\right) A\left(\omega_{0}\right)= & \int K_{\epsilon}\left(\omega, \omega_{1}\right) A\left(\omega_{1}\right) V_{\Delta}\left(\omega_{1}, \omega_{0}\right) \mathrm{d} \omega_{1} A\left(\omega_{0}\right) \\
& +\epsilon^{2} \int K_{\epsilon}\left(\omega, \omega_{2}\right) \int H\left(\omega_{2}-\omega_{1}\right) R\left(\omega_{1}\right) H\left(\omega_{1}-\omega_{0}\right) \mathrm{d} \omega_{1} \mathrm{~d} \omega_{2}, \quad \omega, \omega_{0} \in R^{1} .
\end{aligned}
$$

Hereafter $V_{\Delta}=V_{\Delta_{\epsilon}}\left(\omega_{1}, \omega_{0}\right)=E\left[\xi_{\epsilon}\left(\omega_{1}\right) \xi_{\epsilon}\left(\omega_{0}\right)\right]=D_{\epsilon}^{2} \sum_{i=-l}^{i=l} \chi\left(\omega_{1} \in I_{i}, \omega_{0} \in I_{i}\right)$ with $\omega_{0}, \omega_{1} \in R^{1}$.

In what follows, we shall make use of operator symbolic in the problem of risk minimization. Denote $A, R$ the multiplication operators with the kernels $A(\omega), R(\omega)$. Denote $K_{\epsilon}, V_{\Delta}, H$ the integral operators $L_{2}\left(R^{1}\right) \rightarrow L_{2}\left(R^{1}\right)$ with the kernels $K_{\epsilon}\left(\omega, \omega_{0}\right), V_{\Delta}\left(\omega, \omega_{0}\right), H\left(\omega-\omega_{0}\right)$ respectively and denote $I$ the unit operator. In this notation the Bayes estimator minimizes the Bayes risk

$$
\rho_{\epsilon}(K)=\operatorname{Sp}\left[\left(K^{T} A-I\right) V_{\Delta}(A K-I)+\epsilon^{2} K^{T} H R H K\right]
$$

among all estimators $x_{\epsilon}^{*}=k * y$. Here $\operatorname{Sp}[U]$ denotes the trace of operator $U$ and $K^{T}$ denotes the conjugate operator of $K$.

Denote $R_{\epsilon}(\omega)=R(\omega) \chi\left(|\omega|<\omega_{2 \epsilon}\right)$ and

$$
\bar{R}_{\epsilon}\left(\omega, \omega_{0}\right)=\int H\left(\omega-\omega_{1}\right) R_{\epsilon}\left(\omega_{1}\right) H\left(\omega_{1}-\omega_{0}\right) \mathrm{d} \omega_{1} \chi\left(\omega \in\left(-\omega_{2 \epsilon}, \omega_{2 \epsilon}\right), \omega_{0} \in\left(-\omega_{2 \epsilon}, \omega_{2 \epsilon}\right)\right) .
$$

Since $R_{\epsilon} \leq R$, we get

$$
\begin{aligned}
\bar{\rho}_{\epsilon}(K) & =\operatorname{Sp}\left[\left(K^{T} A-I\right) V_{\Delta}(A K-I)+\epsilon^{2} K^{T} \bar{R}_{\epsilon} K\right] \\
& \leq \operatorname{Sp}\left[\left(K^{T} A-I\right) V_{\Delta}(A K-I)+\epsilon^{2} K^{T} H R_{\epsilon} H K\right] \leq \rho_{\epsilon}(K) .
\end{aligned}
$$

Therefore the problem of obtaining lower bound is reduced to that of minimization of $\bar{\rho}_{\epsilon}(K)$. Define the operator $\bar{K}_{\epsilon}$ such that $\bar{\rho}_{\epsilon}\left(\bar{K}_{\epsilon}\right)=\inf \bar{\rho}_{\epsilon}(K)$ where the infimum is over all operators $K$ such that $\bar{\rho}_{\epsilon}(K)<\infty$.

If we write a version of equation (40) for the operator $\bar{K}_{\epsilon}$, we get easily

$$
\bar{K}_{\epsilon}=V_{\Delta} A\left(A V_{\Delta} A+\epsilon^{2} \bar{R}_{\epsilon}\right)^{-1} .
$$

It follows from the definition of $\bar{\rho}_{\epsilon}(K)$ that in the problem of minimization of $\bar{\rho}_{\epsilon}(K)$ it suffices to consider the operators $A, V_{\Delta}, \bar{R}_{\epsilon}$ as operators $L_{2}\left(\left(-\omega_{2 \epsilon}, \omega_{2 \epsilon}\right)\right) \rightarrow L_{2}\left(\left(-\omega_{2 \epsilon}, \omega_{2 \epsilon}\right)\right)$. Thus, in what follows, we suppose that such a contraction of operators takes place.

For any linear operator $G: L_{2}\left(-\omega_{2 \epsilon}, \omega_{2 \epsilon}\right) \rightarrow L_{2}\left(-\omega_{2 \epsilon}, \omega_{2 \epsilon}\right)$ denote

$$
\|G\|=\sup \left\{x^{\prime} G x:\|x\|=1, x \in L_{2}\left(-\omega_{2 \epsilon}, \omega_{2 \epsilon}\right)\right\}
$$

where $x^{\prime} y$ stands for the inner product of $x, y \in L_{2}\left(-\omega_{2 \epsilon}, \omega_{2 \epsilon}\right)$.

In this notation there holds

$$
\left\|\bar{K}_{\epsilon}\right\| \leq\left\|A^{-1}||\right\| A K_{\epsilon}\|\leq\| A^{-1} \| \leq A^{-1}\left(\omega_{\epsilon}\right) .
$$


We shall use also the following inequality. For any operator $G$ and positive operator $M$ it holds

$$
\operatorname{Sp}[G M] \leq\|G\| \operatorname{Sp}[M]
$$

if $\mathrm{Sp}[M]<\infty$ and $\|G\|<\infty$.

It is known also that, if $G(s, t)$ is the kernel of linear operator $G$, then

$$
\|G\|^{2} \leq \int_{-\omega_{2 \epsilon}}^{\omega_{2 \epsilon}} \int_{-\omega_{2 \epsilon}}^{\omega_{2 \epsilon}} G^{2}(s, t) \mathrm{d} s \mathrm{~d} t .
$$

Substituting (43) in the left-hand side of (42), we get

$$
\begin{aligned}
\bar{\rho}_{\epsilon}\left(\bar{K}_{\epsilon}\right)= & \epsilon^{4} \operatorname{Sp}\left[\left(A V_{\Delta} A+\epsilon^{2} \bar{R}_{\epsilon}\right)^{-1} \bar{R}_{\epsilon} V_{\Delta} \bar{R}_{\epsilon}\left(A V_{\Delta} A+\epsilon^{2} \bar{R}_{\epsilon}\right)^{-1}\right] \\
& +\epsilon^{2} \operatorname{Sp}\left[\left(A V_{\Delta} A+\epsilon^{2} \bar{R}_{\epsilon}\right)^{-1} A V_{\Delta} \bar{R}_{\epsilon} V_{\Delta} A\left(A V_{\Delta} A+\epsilon^{2} \bar{R}_{\epsilon}\right)^{-1}\right] .
\end{aligned}
$$

Now we define some discrete version $\bar{\rho}_{\epsilon \Delta}\left(\bar{K}_{\epsilon}\right)$ of $\bar{\rho}_{\epsilon}\left(\bar{K}_{\epsilon}\right)$ and show that $\left|\bar{\rho}_{\epsilon \Delta}\left(\bar{K}_{\epsilon}\right)-\bar{\rho}_{\epsilon}\left(K_{\epsilon}\right)\right|=o\left(\bar{\rho}_{\epsilon \Delta}\left(\bar{K}_{\epsilon}\right)\right)$ is negligible. After that we show that $\bar{\Psi}_{\epsilon}\left(\omega_{\epsilon}\right)$ is the lower bound of $\bar{\rho}_{\epsilon \Delta}\left(\bar{K}_{\epsilon}\right)$. The proofs of these statements have technical character and are based on inequalities (44)-(46).

For each $i,|i| \leq l$ we fix $\omega_{i} \in I_{i}$ and define the functions

$$
\begin{gathered}
A_{\Delta}(\omega)=\sum_{i=-l}^{l} A\left(\omega_{i}\right) \chi\left(\omega \in I_{i}\right), \\
H_{\Delta}\left(\omega, \omega_{1}\right)=\sum_{i=-l}^{l} H\left(\omega_{i}-\omega_{1}\right) \chi\left(\omega \in I_{i}\right) . \\
R_{\Delta}\left(\omega_{0}, \omega_{1}\right)=\left(H_{\Delta}^{T} R_{\epsilon} H_{\Delta}\right)\left(\omega_{0}, \omega_{1}\right) .
\end{gathered}
$$

Denote

We have

$$
\bar{\rho}_{\epsilon \Delta}\left(\bar{K}_{\epsilon}\right)=\operatorname{Sp}\left[\left(\bar{K}_{\epsilon}^{T} A_{\Delta}-I\right) V_{\Delta}\left(A_{\Delta} \bar{K}_{\epsilon}-I\right)+\epsilon^{2} \bar{K}_{\epsilon}^{T} R_{\Delta} \bar{K}_{\epsilon}\right] .
$$

$$
\begin{aligned}
\bar{\rho}_{\epsilon}\left(\bar{K}_{\epsilon}\right)-\bar{\rho}_{\epsilon \Delta}\left(\bar{K}_{\epsilon}\right)= & \operatorname{Sp}\left[\bar{K}_{\epsilon}^{T}\left(A-A_{\Delta}\right) V_{\Delta}\left(A \bar{K}_{\epsilon}-I\right)\right] \\
& +\operatorname{Sp}\left[\bar{K}_{\epsilon}^{T} A_{\Delta} V_{\Delta}\left(A-A_{\Delta}\right) \bar{K}_{\epsilon}\right]+\epsilon^{2} \operatorname{Sp}\left[\bar{K}_{\epsilon}^{T}\left(\bar{R}_{\epsilon}-R_{\Delta}\right) \bar{K}_{\epsilon}\right] \doteq J_{1}+J_{2}+\epsilon^{2} J_{3} .
\end{aligned}
$$

Since $\left\|A \bar{K}_{\epsilon}-I\right\| \leq 1$ and $\left\|\bar{K}_{\epsilon} A\right\| \leq 1$, by A4, (44), we get

$$
\left|J_{1}\right| \leq A^{-1}\left(\omega_{\epsilon}\right)\left\|A \bar{K}_{\epsilon}\right\|\left\|A-A_{\Delta}\right\| \operatorname{Sp}\left[V_{\Delta}\right]\left\|A \bar{K}_{\epsilon}-I\right\| \leq C A^{-1}\left(\omega_{\epsilon}\right) \Delta \operatorname{Sp}\left[V_{\Delta}\right] .
$$

Hereafter $C$ stands for positive constants.

Arguing similarly, we get

$$
\left|J_{2}\right| \leq A^{-1}\left(\omega_{\epsilon}\right)\left\|A \bar{K}_{\epsilon}||\right\| A_{\Delta}\left\|\operatorname{Sp}\left[V_{\Delta}\right]\right\| A-A_{\Delta}\left\|A^{-1}\left(\omega_{\epsilon}\right)\right\| A \bar{K}_{\epsilon} \| \leq C \Delta A^{-2}\left(\omega_{\epsilon}\right) \operatorname{Sp}\left[V_{\Delta}\right] .
$$

We have

$$
J_{3}=\operatorname{Sp}\left[\bar{K}_{\epsilon} \bar{K}_{\epsilon}^{T}\left(H-H_{\Delta}\right) R_{\epsilon} H\right]+\operatorname{Sp}\left[\bar{K}_{\epsilon} \bar{K}_{\epsilon}^{T} H_{\Delta} R_{\epsilon}\left(H-H_{\Delta}\right)\right] \doteq J_{31}+J_{32} .
$$

By H3, the function $H(\omega)$ satisfies the Lipschitz conditions. Hence, using (46), we get

$$
\left\|H-H_{\Delta}\right\| \leq\left(\int_{-\omega_{\epsilon}}^{\omega_{\epsilon}} \mathrm{d} \omega_{0} \int_{-\omega_{\epsilon}}^{\omega_{\epsilon}} \mathrm{d} \omega\left|H\left(\omega_{0}-\omega\right) \sum_{i=-l}^{l} H\left(\omega_{i}-\omega\right) \chi\left(\omega_{0} \in I_{i}\right)\right|^{2}\right)^{1 / 2} \leq C \Delta \omega_{\epsilon}
$$


and

$$
\begin{aligned}
\left\|H_{\Delta}\right\| & \leq\left(\int_{-\omega_{\epsilon}}^{\omega_{\epsilon}} \mathrm{d} \omega_{0} \int_{-\omega_{\epsilon}}^{\omega_{\epsilon}} \mathrm{d} \omega\left|\sum_{i=-l}^{l} H\left(\omega_{i}-\omega\right) \chi\left(\omega_{0} \in I_{i}\right)\right|^{2}\right)^{1 / 2} \\
& =\left(\int_{-\omega_{\epsilon}}^{\omega_{\epsilon}} \mathrm{d} \omega_{0} \int H^{2}\left(\omega_{0}-\omega\right) \mathrm{d} \omega\right)^{1 / 2}(1+o(1)) \leq C \omega_{\epsilon}^{1 / 2}\|H\| .
\end{aligned}
$$

Hence, using (44), (45), we get

$$
\begin{gathered}
J_{31} \leq\left\|K_{\epsilon}\right\|^{2}\left\|H-H_{\Delta}\right\| \operatorname{Sp}\left[R_{\epsilon}\right]\|H\| \leq C \Delta \omega_{\epsilon} A^{-2}\left(\omega_{2 \epsilon}\right) \operatorname{Sp}\left[R_{\epsilon}\right], \\
I_{32} \leq\left\|K_{\epsilon}\right\|^{2}\left\|H_{\Delta}\right\| \operatorname{Sp}\left[R_{\epsilon}\right]\left\|H-H_{\Delta}\right\| \leq C \Delta \omega_{\epsilon}^{3 / 2} A^{-2}\left(\omega_{2 \epsilon}\right) \operatorname{Sp}\left[R_{\epsilon}\right] .
\end{gathered}
$$

By definition (37) of $\hat{\xi}_{\epsilon}(\omega)$, we get

$$
\operatorname{Sp}\left[V_{\Delta}\right]=o\left(D_{\epsilon}^{2} \omega_{\epsilon}\right)
$$

and, by R, we get

$$
\operatorname{Sp}\left[R_{\epsilon}\right]=O\left(\omega_{\epsilon}\right)
$$

By (49)-(52), (56)-(58), we get

$$
\begin{aligned}
\left|\bar{\rho}_{\epsilon}\left(\bar{K}_{\epsilon}\right)-\bar{\rho}_{\epsilon}\left(\bar{K}_{\epsilon}\right)\right| & \leq C \Delta A^{-2}\left(\omega_{\epsilon}\right) \operatorname{Sp}\left[V_{\Delta}\right]+C \epsilon^{2} \Delta \omega_{\epsilon}^{3 / 2} A^{-2}\left(\omega_{\epsilon}\right) \operatorname{Sp}\left[R_{\epsilon}\right] \\
& \leq C \Delta A^{-2}\left(\omega_{\epsilon}\right) D_{\epsilon}^{2} \omega_{\epsilon}+C \epsilon^{2} \Delta \omega_{\epsilon}^{5 / 2} A^{-2}\left(\omega_{\epsilon}\right) .
\end{aligned}
$$

By (36), (59), using $\bar{\Psi}_{\epsilon}\left(\omega_{\epsilon}\right)=O\left(\epsilon^{2} A^{-2}\left(\omega_{\epsilon}\right) R\left(\omega_{\epsilon}\right) \omega_{\epsilon}\right)$, we get

$$
\bar{\rho}_{\epsilon}\left(\bar{K}_{\epsilon}\right)-\rho_{\epsilon \Delta}\left(\bar{K}_{\epsilon}\right)=o\left(\bar{\Psi}\left(\omega_{\epsilon}\right)\right) .
$$

It remains to study the problem of minimization of $\bar{\rho}_{\epsilon \Delta}(K)$. In discrete setting, $V_{\Delta}=D_{\epsilon}^{2} I$. This simplifies essentially estimates.

The next two estimates are auxiliary. By (54), we get

$$
\left\|R_{\Delta}\right\| \leq\left\|H_{\Delta}\right\|^{2}\left\|R_{\epsilon}\right\| \leq C \omega_{\epsilon} .
$$

Since $\left\|A_{\Delta}^{-1}\right\| \leq A^{-1}\left(\omega_{\epsilon}\right)$, we have also

$$
\left\|A_{\Delta}^{-1} R_{\Delta} A_{\Delta}^{-1}\right\| \leq A^{-2}\left(\omega_{\epsilon}\right)\left\|R_{\Delta}\right\| \leq C A^{-2}\left(\omega_{\epsilon}\right) \omega_{\epsilon} .
$$

Now we utilize (61), (62), to estimate

$$
\begin{aligned}
\hat{\rho}_{\epsilon \Delta}= & \inf _{K} \bar{\rho}_{\epsilon \Delta}(K)=\epsilon^{4} \operatorname{Sp}\left[\left(A_{\Delta} V_{\Delta} A_{\Delta}+\epsilon^{2} R_{\Delta}\right)^{-1} R_{\Delta} V_{\Delta} R_{\Delta}\left(A_{\Delta} V_{\Delta} A_{\Delta}+\epsilon^{2} R_{\Delta}\right)^{-1}\right] \\
& +\epsilon^{2} \operatorname{Sp}\left[\left(A_{\Delta} V_{\Delta} A_{\Delta}+\epsilon^{2} R_{\Delta}\right)^{-1} A_{\Delta} V_{\Delta} R_{\Delta} V_{\Delta} A_{\Delta}\left(A_{\Delta} V_{\Delta} A_{\Delta}+\epsilon^{2} R_{\Delta}\right)^{-1}\right] \doteq U_{1}+U_{2} .
\end{aligned}
$$

We have

$$
\begin{aligned}
U_{1} \leq & \epsilon^{4} D_{\epsilon}^{-4} A^{-4}\left(\omega_{2 \epsilon}\right) \operatorname{Sp}\left[\left(I+\epsilon^{2} D_{\epsilon}^{-1} A_{\Delta}^{-1} R_{\Delta} A_{\Delta}^{-1}\right)^{-1}\right. \\
& \left.\times R_{\Delta} V_{\Delta} R_{\Delta}\left(I+\epsilon^{2} D_{\epsilon}^{-1} A_{\Delta}^{-1} R_{\Delta} A_{\Delta}^{-1}\right)^{-1}\right] \\
\leq & C \epsilon^{4} D_{\epsilon}^{-4} A^{-4}\left(\omega_{2 \epsilon}\right)\left\|R_{\Delta}\right\|^{2} \operatorname{Sp}\left[V_{\Delta}\right] \leq C \epsilon^{4} D_{\epsilon}^{-2} A^{-4}\left(\omega_{\epsilon}\right)\left\|R_{\Delta}\right\|^{2} \omega_{\epsilon} \\
\leq & C \epsilon^{4} D_{\epsilon}^{-2} A^{-4}\left(\omega_{2 \epsilon}\right) \omega_{\epsilon}^{3}=o\left(\epsilon^{2} \operatorname{Sp}\left[A^{-2} R\right]\right)
\end{aligned}
$$

where the last equality follows from (34), (35). 
We have

$$
U_{2}=U_{21}-U_{22}-U_{23}
$$

where

$$
\begin{aligned}
U_{21} & =\epsilon^{2} \operatorname{Sp}\left[A_{\Delta}^{-1} R_{\Delta} A_{\Delta}^{-1}\right], \\
U_{22} & =\epsilon^{4} \operatorname{Sp}\left[\left(A_{\Delta} V_{\Delta} A_{\Delta}+\epsilon^{2} R_{\Delta}\right)^{-1} R_{\Delta} A_{\Delta}^{-1} R_{\Delta} A_{\Delta}^{-1} A_{\Delta} V_{\Delta} A_{\Delta}\left(A_{\Delta} V_{\Delta} A_{\Delta}+\epsilon^{2} R_{\Delta}\right)^{-1}\right], \\
U_{23} & =\epsilon^{4} \operatorname{Sp}\left[A^{-1} R_{\Delta} A^{-1} R_{\Delta}\left(A_{\Delta} V_{\Delta} A_{\Delta}+\epsilon^{2} R_{\Delta}\right)^{-1}\right] .
\end{aligned}
$$

The estimates $U_{22}, U_{23}$ are akin to (50) and (64)

$$
\begin{gathered}
U_{22} \leq \epsilon^{4} D_{\epsilon}^{-2} A^{-2}\left(\omega_{2 \epsilon}\right)\left\|R_{\Delta}\right\| \operatorname{Sp}\left[A_{\Delta}^{-1} R_{\Delta} A_{\Delta}^{-1}\right]\left\|A_{\Delta} V_{\Delta} A_{\Delta}\left(A_{\Delta} V_{\Delta} A_{\Delta}+\epsilon^{2} R_{\Delta}\right)^{-1}\right\| \\
\leq C \epsilon^{4} D_{\epsilon}^{-2} A^{-2}\left(\omega_{2 \epsilon}\right) \omega_{\epsilon} \operatorname{Sp}\left[A_{\Delta}^{-2} R_{\epsilon}\right](1+o(1))=o\left(\epsilon^{2} \operatorname{Sp}\left[A_{\Delta}^{-2} R_{\epsilon}\right]\right), \\
U_{23} \leq \epsilon^{4}\left\|R_{\Delta}\left(A_{\Delta} V_{\Delta} A_{\Delta}+\epsilon^{2} R_{\Delta}\right)^{-1}\right\| \operatorname{Sp}\left[A_{\Delta}^{-2} R_{\Delta}\right] \\
\leq C \epsilon^{4}\left\|R_{\Delta}\right\| A^{-2}\left(\omega_{2 \epsilon}\right) D_{\epsilon}^{-2} \operatorname{Sp}\left[A_{\Delta}^{-2} R_{\Delta}\right] \\
\leq C \epsilon^{4} \omega_{\epsilon} A^{-2}\left(\omega_{2 \epsilon}\right) D_{\epsilon}^{-2} \operatorname{Sp}\left[A_{\Delta}^{-2} R_{\Delta}\right]=o\left(\epsilon^{2} \operatorname{Sp}\left[A_{\Delta}^{-2} R_{\Delta}\right]\right)
\end{gathered}
$$

where the last equalities in (66), (67) follows from (34), (35).

Now (63)-(67) together imply

$$
\hat{\rho}_{\epsilon \Delta}=\epsilon^{2} \operatorname{Sp}\left[A_{\Delta}^{-2} R_{\Delta}\right](1+o(1)) .
$$

This completes the proof of lower bound in (12).

\subsection{Proof of Theorem $\mathbf{1 . 2}$}

We begin with the proof of lower bound. The arguments are akin to the proof of Theorem 1.2 in [11] and are based on the method proposed in [8]. Define the parametric family of functions

$$
G_{\theta}(\omega)=\frac{1}{\sqrt{2}} \tilde{\delta}_{\epsilon}^{-1 / 2} \theta \chi\left(\omega_{\epsilon}<|\omega|<\left(1+\tilde{\delta}_{\epsilon}\right) \omega_{\epsilon}\right)=\theta \tilde{H}_{\epsilon}
$$

where $\tilde{\delta}_{\epsilon} \asymp \omega_{\epsilon}^{1-\beta-\kappa}, 0<\kappa<\gamma-\beta$.

Consider the problem of estimation of parameter $\theta$ if $\theta$ has the Binomial distribution

$$
P\left(\theta= \pm \theta_{\epsilon}\right)=\frac{1}{2}, \quad \theta_{\epsilon}=B^{-1}\left(\omega_{\epsilon}\right) .
$$

Since the noise is Gaussian it is easy to find the sufficient statistics in this problem. As a result the problem is reduced to the estimation of $\theta$ on observation

$$
y_{\epsilon}=\theta+\epsilon\left\|\tilde{H}_{\epsilon}\right\|^{-1} \int \tilde{H}_{\epsilon}(\omega) A^{-1}(\omega) \int H\left(\omega-\omega_{1}\right) R^{1 / 2}\left(\omega_{1}\right) \mathrm{d} w\left(\omega_{1}\right)
$$

that can be written in a more simple form

$$
y_{\epsilon}=\theta+d_{\epsilon} \zeta
$$

where $\zeta$ is Gaussian random variable, $E \zeta=0, E \zeta^{2}=1$ and

$$
d_{\epsilon}^{2}=\epsilon^{2}\left\|\tilde{H}_{\epsilon}\right\|^{-2} \int R\left(\omega_{1}\right)\left(\int H\left(\omega_{1}-\omega\right) A^{-1}(\omega) \tilde{H}_{\epsilon}(\omega) \mathrm{d} \omega\right)^{2} \geq C\|H\|^{2} \tilde{\delta}_{\epsilon} R\left(\omega_{\epsilon}\right) A^{-2}\left(\omega_{\epsilon}\right) .
$$


Since $\theta_{\epsilon}=o\left(d_{\epsilon}\right)$, the Bayes risks equals

$$
\theta_{\epsilon}^{2}(1+o(1))=B^{-2}\left(\omega_{\epsilon}\right)(1+o(1))
$$

and this is the lower bound of minimax risks.

The proof of upper bounds is also akin to that of Theorem 1.2 in [11] and is omitted.

\subsection{Proof of Theorem 1.3. lower bound}

We consider the auxiliary problem of Bayes estimation with spectral density $\bar{V}_{\epsilon}(\omega) \leq V(\omega), \omega \in R^{1}$ such that the reasoning the proof of Theorem 1.3 in [11] can be applied. As a result we get the required lower bound in Theorem 1.3.

We put $\bar{V}_{\epsilon}(\omega)=0$ if $|\omega|>\left(1-\bar{\delta}_{\epsilon}\right) \omega_{1 \epsilon} \doteq \bar{\omega}_{\epsilon}$ and $\bar{V}_{\epsilon}(\omega)=V\left(\bar{\omega}_{\epsilon}\right)$ if $|\omega|<\bar{\omega}_{\epsilon}$ where $\bar{\omega}_{\epsilon}$ is such that

$$
\epsilon^{2} A^{-2}\left(\omega_{\epsilon}\right) R\left(\omega_{\epsilon}\right)=o\left(V\left(\bar{\omega}_{\epsilon}\right)\right)
$$

and $\delta_{\epsilon} \rightarrow 0$ as $\epsilon \rightarrow 0$.

Since

$$
\begin{aligned}
\rho_{\epsilon}(K) & =\operatorname{Sp}\left[\left(K^{T} A-I\right) H V H(A K-I)+\epsilon^{2} K^{T} H R H K\right] \\
& \geq \operatorname{Sp}\left[\left(K^{T} A-I\right) H \bar{V}_{\epsilon} H\left(A K_{I}\right)+\epsilon^{2} K^{T} H R H K\right] \doteq \hat{\rho}_{\epsilon}(K)
\end{aligned}
$$

it suffices to find lower bound for the asymptotic of $\hat{\rho}_{\epsilon}(K)$. Such a lower bound can be found if we repeat all estimates in the proof of lower bound for the asymptotic of Bayes risks in Theorem 1.3 in [11]. This lower bound equals

$$
\epsilon^{2}\|h\|^{2}\left\|h_{1}\right\|^{2} \int \frac{R(\omega) \bar{V}_{\epsilon}(\omega)}{\left\|h_{1}\right\|^{2} A^{2}(\omega) \bar{V}_{\epsilon}(\omega)+\epsilon^{2}\|h\|^{2} R(\omega)} \mathrm{d} \omega(1+o(1))=\epsilon^{2}\|h\|^{2} \int_{-\omega_{\epsilon}}^{\omega_{\epsilon}} A^{-2}(\omega) R(\omega) \mathrm{d} \omega(1+o(1))
$$

where the last relation follows from (68). This completes the proof of lower bound in Theorem 1.3.

\subsection{Proof of Theorem 1.1. Upper bound}

The proofs of upper bounds differ from the standard reasoning (see $[8,10,26]$ ) only by the presence of the function $h$ in the noise. Thus the main difference in the reasoning is the following. One needs to show that the Fourier transform of correlation function of $\epsilon h(t) \zeta(t)$ given by the convolution

$$
\epsilon^{2} \int H^{2}\left(\omega-\omega_{1}\right) R\left(\omega_{1}\right) \mathrm{d} \omega_{1}
$$

can be replaced the simple asymptotic $\epsilon^{2}\|h\|^{2} R(\omega)(1+o(1))$ for the large values of $\omega$. By H2, $R$, the function $H(\omega)$ decreases faster then $R(\omega)$ and the function $R(\omega)$ is regularly varying. This allows to utilize the required asymptotic in our estimates.

We have

$$
\begin{aligned}
\rho_{\epsilon}\left(x_{\omega_{\epsilon}}^{* *}\right) & =\sup _{x \in Q} \int X^{2}(\omega) \chi\left(|\omega|>\omega_{\epsilon}\right) \mathrm{d} \omega+\epsilon^{2} \int_{-\omega_{\epsilon}}^{\omega_{\epsilon}} A^{-2}(\omega) \int H^{2}\left(\omega-\omega_{1}\right) R\left(\omega_{1}\right) \mathrm{d} \omega_{1} \mathrm{~d} \omega \\
& =o\left(B^{-2}\left(\omega_{\epsilon}\right)\right)+\bar{\Psi}_{\epsilon}\left(\omega_{\epsilon}\right)(1+o(1))=\bar{\Psi}_{\epsilon}\left(\omega_{\epsilon}\right)(1+o(1)) .
\end{aligned}
$$

This implies the upper bound for the estimator $x_{\omega_{\epsilon}}^{* *}$.

By the definition of $\mu_{\epsilon}$, it is easy to see that $\Psi_{\epsilon}\left(\mu_{\epsilon}\right)=\bar{\Psi}_{\epsilon}\left(\omega_{\epsilon}\right)(1+o(1))$. 
The minimax risk of Tikhonov regularizing procedure equals

$$
\begin{aligned}
\rho_{\epsilon}\left(x_{\alpha_{\epsilon}}^{* *}\right)= & \alpha_{\epsilon}^{2} \max _{\omega} M^{2}(\omega) B^{-2}(\omega)\left(A^{2}(\omega)+\alpha_{\epsilon} M(\omega)\right)^{-2} \\
& +\epsilon^{2} \int A^{2}(\omega)\left(A^{2}(\omega)+\alpha_{\epsilon} M(\omega)\right)^{-2} \int H^{2}\left(\omega-\omega_{1}\right) R\left(\omega_{1}\right) \mathrm{d} \omega_{1} \mathrm{~d} \omega \doteq I_{1}+I_{2} .
\end{aligned}
$$

By $\mathrm{H} 2, R$, we get

$$
I_{2}=2 \epsilon^{2}\|h\|^{2} \int_{\delta \omega_{\epsilon}}^{\infty} A^{2}(\omega) R(\omega)\left(A^{2}(\omega)+\alpha_{\epsilon} M(\omega)\right)^{-2} \mathrm{~d} \omega+O\left(\epsilon^{2} \delta \omega_{\epsilon} A^{-2}\left(\delta \omega_{\epsilon}\right) R\left(\delta \omega_{\epsilon}\right)\right)
$$

for any $0<\delta<1$.

Define $\delta_{\epsilon}$ such that $A^{2}\left(\omega_{\epsilon}\right)=A^{2}\left(\omega_{\epsilon}\left(1-\delta_{\epsilon}\right)\right)(1+o(1))$ and $M\left(\omega_{\epsilon}\left(1-\delta_{\epsilon}\right)\right)=o\left(M\left(\omega_{\epsilon}\right)\right)$. Then

$$
I_{2}=I_{21}+I_{22}+I_{23}+O\left(\epsilon^{2} \delta \omega_{\epsilon} A^{-2}\left(\delta \omega_{\epsilon}\right) R\left(\delta \omega_{\epsilon}\right)\right)
$$

where

$$
\begin{aligned}
I_{21} & =2 \epsilon^{2}\|h\|^{2} \int_{\delta \omega_{\epsilon}}^{\left(1-\delta_{\epsilon}\right) \omega_{\epsilon}} A^{2}(\omega) R(\omega)\left(A^{2}(\omega)+\alpha_{\epsilon} M(\omega)\right)^{-2} \mathrm{~d} \omega \\
& =2 \epsilon^{2}\|h\|^{2} \int_{\delta \omega_{\epsilon}}^{\left(1-\delta_{\epsilon}\right) \omega_{\epsilon}} A^{-2}(\omega) R(\omega) \mathrm{d} \omega(1+o(1))=\bar{\Psi}_{\epsilon}\left(\omega_{\epsilon}\right)(1+o(1)), \\
I_{22} & =2 \epsilon^{2}\|h\|^{2} \int_{\left(1-\delta_{\epsilon}\right) \omega_{\epsilon}}^{\left(1+\delta_{\epsilon}\right) \omega_{\epsilon}} A^{2}(\omega) R(\omega)\left(A^{2}(\omega)+\alpha_{\epsilon} M(\omega)\right)^{-2} \mathrm{~d} \omega, \\
I_{23} & =2 \epsilon^{2}\|h\|^{2} \int_{\left(1+\delta_{\epsilon}\right) \omega_{\epsilon}}^{\infty} A^{2}(\omega) R(\omega)\left(A^{2}(\omega)+\alpha_{\epsilon} M(\omega)\right)^{-2} \mathrm{~d} \omega .
\end{aligned}
$$

By M2 and the definitions of $\delta_{\epsilon}, \omega_{\epsilon}$, we get

$$
I_{22}=o\left(I_{21}\right), I_{23}=o\left(I_{21}\right)
$$

By (72)-(74), we get

$$
I_{2}=I_{21}(1+o(1))=\bar{\Psi}_{\epsilon}\left(\omega_{\epsilon}\right)(1+o(1))
$$

By M2 and the definition of $\alpha_{\epsilon}$, we get

$$
I_{1}<C B^{-2}\left(\omega_{\epsilon}\right)=o\left(\bar{\Psi}_{\epsilon}\left(\omega_{\epsilon}\right)\right)
$$

Now (70), (75), (76) together imply the asymptotic minimaxity of Tikhonov regularizing procedure.

\subsection{Proof of Theorem 1.3. Upper bound}

The Bayes risk equals

$$
2 \int_{\omega_{\epsilon}}^{\infty} \int H_{1}^{2}\left(\omega-\omega_{1}\right) V\left(\omega_{1}\right) \mathrm{d} \omega \mathrm{d} \omega_{1}+\epsilon^{2} \int_{-\omega_{\epsilon}}^{\omega_{\epsilon}} A^{-2}(\omega) \int H^{2}\left(\omega-\omega_{1}\right) R\left(\omega_{1}\right) \mathrm{d} \omega_{1} \doteq 2 I_{1}+I_{2} .
$$


By straightforward calculations, we get

$$
I_{2}=\epsilon^{2}\|H\|^{2} \int_{-\omega_{\epsilon}}^{\omega_{\epsilon}} A^{-2}(\omega) R(\omega) \mathrm{d} \omega(1+o(1)) .
$$

Denote $\omega_{2 \epsilon}=\frac{1}{2} \omega_{1 \epsilon}+\frac{1}{2} \omega_{\epsilon}$.

We have

$$
I_{1}=I_{11}+I_{12}
$$

where

$$
\begin{aligned}
& I_{11}=\int_{\omega_{\epsilon}}^{\infty} \int_{\omega_{2 \epsilon}}^{\infty} H_{1}^{2}\left(\omega-\omega_{1}\right) V\left(\omega_{1}\right) \mathrm{d} \omega \mathrm{d} \omega_{1}, \\
& I_{12}=\int_{\omega_{\epsilon}}^{\infty} \int_{-\infty}^{\omega_{2 \epsilon}} H_{1}^{2}\left(\omega-\omega_{1}\right) V\left(\omega_{1}\right) \mathrm{d} \omega \mathrm{d} \omega_{1} .
\end{aligned}
$$

By (50), (30), we get

$$
\begin{aligned}
I_{11} & \leq \int_{\omega_{\epsilon}}^{\infty} \int_{\omega_{2 \epsilon}}^{\infty} H_{1}^{2}\left(\omega-\omega_{1}\right) V\left(\omega_{1}\right) \mathrm{d} \omega \mathrm{d} \omega_{1} \\
& \leq\left\|H_{1}\right\|^{2} \int_{\omega_{2 \epsilon}}^{\infty} V\left(\omega_{1}\right) \mathrm{d} \omega_{1}=o\left(I_{2}\right), \\
I_{12} & \leq \int_{\omega_{2 \epsilon}-\omega_{\epsilon}}^{\infty} H_{1}^{2}(\omega) \mathrm{d} \omega \int V(\omega) \mathrm{d} \omega=o\left(I_{1}\right) .
\end{aligned}
$$

Now the upper bound follows from (77)-(83).

Acknowledgements. The author thanks referees and associate editor for helpful comments and remarks.

\section{REFERENCES}

[1] L.D. Brown, T. Cai, M.G. Low and C. Zang, Asymptotic equivalence theory for nonparametric regression with random design. Ann. Stat. 24 (2002) 2399-2430.

[2] C. Butucea, Deconvolution of supersmooth densities with smooth noise. Canad. J. Statist. 32 (2004) 181-192.

[3] C. Butucea and A.B. Tsybakov, Sharp optimality for density deconvolution with dominating bias. (2004), arXiv: math.ST/0409471.

[4] L. Cavalier, G.K. Golubev, O.V. Lepski and A.B. Tsybakov, Block thresholding and sharp adaptive estimation in severely ill-posed problems. Theory Probab. Appl. 48 (2003) 534-556.

[5] G.K. Golubev and R.Z. Khasminskii, Statistical approach to Cauchy problem for Laplace equation. State of the Art in Probability and Statistics, Festschrift for W.R. van, Zwet M. de Gunst, C. Klaassen and van der Vaart Eds., IMS Lecture Notes Monograph Series 36 (2001) 419-433.

[6] R.J. Carrol and P. Hall, Optimal rates of convergence for deconvolving a density J. Amer. Statist. Assoc. 83 (1988) $1184-1186$.

[7] D.L. Donoho, Nonlinear solution of linear inverse problems by wavelet-vaguelette decomposition. Appl. Comput. Harmon. Anal. 2 (1992) 101-126.

[8] S. Efroimovich, Nonparametric Curve Estimation: Methods, Theory and Applications. New York, Springer (1999).

[9] S. Efromovich and M. Pinsker, Sharp optimal and adaptive estimation for heteroscedastic nonparametric regression. Statistica Cinica 6 (1996) 925-942.

[10] M.S. Ermakov, Minimax estimation in a deconvolution problem. J. Phys. A: Math. Gen. 25 (1992) 1273-1282.

[11] M.S. Ermakov, Asymptotically minimax and Bayes estimation in a deconvolution problem. Inverse Problems 19 (2003) 1339 1359.

[12] J. Fan, Asymptotic normality for deconvolution kernel estimators. Sankhia Ser. A 53 (1991) 97-110.

[13] J. Fan, On the optimal rates of convergence for nonparametric deconvolution problems. Ann. Statist. 19 (1991) 1257-1272.

[14] A. Goldenshluger, On pointwise adaptive nonparametric deconvolution. Bernoulli 5 (1999) 907-25. 
[15] Yu K. Golubev, B.Y. Levit and A.B. Tsybakov, Asymptotically efficient estimation of Analitic functions in Gaussian noise. Bernoulli 2 (1996) 167-181.

[16] I.A. Ibragimov and R.Z. Hasminskii, Estimation of distribution density belonging to a class of entire functions. Theory Probab. Appl. 27 (1982) 551-562.

[17] P.A. Jansson, Deconvolution, with application to Spectroscopy. New York, Academic (1984).

[18] I.M. Johnstone, G. Kerkyacharian, D. Picard and M.Raimondo, Wavelet deconvolution in a periodic setting. J. Roy. Stat. Soc. Ser B. 66 (2004) 547-573.

[19] I.M. Johnstone and M. Raimondo, Periodic boxcar deconvolution and Diophantine approximation. Ann. Statist. 32 (2004) $1781-1805$

[20] J. Kalifa and S. Mallat, Threshholding estimators for linear inverse problems and deconvolutions. Ann. Stat. 31 (2003) 58-109.

[21] S. Kassam and H. Poor, Robust techniques for signal processing. A survey. Proc. IEEE 73 (1985) 433-481.

[22] M.R. Leadbetter, G. Lindgren and H. Rootzen, Extremes and Related Properties of Random sequences and Processes. SpringerVerlag NY (1986).

[23] R. Neelamani, H. Choi, R.G. Baraniuk, ForWaRD: Fourier-wavelet regularized deconvolution for ill-conditioned systems. IEEE Trans. Signal Process. 52 (2004) 418-433.

[24] M. Nussbaum, Asymptotic equivalence of density estimation and Gaussian white noise. Ann. Stat. 24 (1996) $2399-2430$.

[25] M. Pensky and B. Vidakovic, Adaptive wavelet estimator for nonparametric density deconvolution. Ann. Statist. 27 (1999) 2033-2053.

[26] M.S. Pinsker, Optimal filtration of square-integral signal in Gaussian noise. Problems Inform. Transm. 16 (1980) 52-68.

[27] M. Schipper, Optimal rates and constants in $L_{2}$-minimax estimation of probability density functions. Math. Methods Stat. 5 (1996) 253-274.

[28] A.J. Smola, B. Scholkopf and K. Miller, The connection between regularization operators and support vector kernels. Newral Networks 11 (1998) 637-649.

[29] A. Tikhonov and V. Arsenin, Solution of Ill-Posed Problems. New-York, Wiley (1977).

[30] A.B. Tsybakov, On the best rate of adaptive estimation in some inverse problems. C.R. Acad. Sci. Paris, Serie 1330 (2000) 835-840.

[31] N. Wiener, Extrapolation, Interpolation and Smoothing of Stationary Time Series. New York, Wiley (1950). 